\title{
Hemodynamic monitoring during surgeries in beach chair position: What can a big picture teach us?
}

\author{
Markus M. Luedi ${ }^{1}$ Karim Bendjelid ${ }^{2}$
}

Received: 19 October 2016/ Accepted: 19 October 2016/Published online: 31 October 2016

(C) Springer Science+Business Media Dordrecht 2016

Of the 26 Million surgeries listed in the U.S. National Anesthesia Clinical Outcomes Registry (NACOR) from 2010 to 2015, Total Shoulder Arthroplasty (TSA), a frequently performed procedure, was listed 38,147 times. Patients undergoing TSA are usually placed in the beach chair position, which improves surgical conditions but at the price of decreased cerebral blood flow. Textbooks describe cerebral blood flow auto regulation as functioning with mean arterial pressure (MAP) $50-150 \mathrm{~mm} \mathrm{Hg}$. Problematically and widely unknown, the lower limit of $50 \mathrm{~mm} \mathrm{Hg}$ for cerebral blood flow auto regulation is based on an investigation in pregnant women published in 1953 even if pregnancy is well known as a vasoplegic state. To decrease blood pressure, the subjects in this $60+$ year old study received hydralazine, a cerebral vasodilator [1, 2]. Interestingly, only 1 year later, Moyer et al. reported symptoms of cerebral ischemia occurring at a MAP of $55 \mathrm{~mm} \mathrm{Hg}$ [3]; nonetheless, $50 \mathrm{~mm} \mathrm{Hg}$ became the standard reported value for the lower limit of cerebral blood flow auto regulation.

No guidelines currently exist for invasive perioperative blood pressure monitoring in patients undergoing TSA in the beach chair position. In this issue, Gabriel

Markus M. Luedi

markus.luedi2@insel.ch

Karim Bendjelid

karim.bendjelid@hcuge.ch

1 Department of Anaesthesiology, Bern University Hospital Inselspital, University of Bern, Freiburgstrasse, 3010 Bern, Switzerland

2 Department of Anaesthesiology, Pharmacology, and Intensive Care, Geneva University Hospitals, Geneva, Switzerland et al. [4] describe patient, surgical, and institutional factors associated with the perioperative installation of arterial lines for blood pressure monitoring during TSA to allow anaesthesiologists to "benchmark" with national norms. They studied 23,073 patients from the U.S. NACOR, and modelled the data in univariable and multivariable logistic regression models to describe factors associated with invasive blood pressure monitoring during TSA in the United States. They found that of all these patients undergoing TSA under general anaesthesia, only $443(1.92 \%)$ had an intra-arterial blood pressure monitoring [4]. The present choice of invasive monitoring was found related to both institutional and patient factors [4]. In this regard, patient age over 65 years, congestive heart failure and surgery lasting at least $180 \mathrm{~min}$ were all associated with increased use for arterial line placement [4]. And compared to university hospitals, arterial line placement was more likely used in attached or freestanding surgical centres and less likely in other hospitals and facilities performing less than 100 TSAs per year [4].

Studying a much smaller cohort, Yadeau et al. recently described patients undergoing TSA with regional anaesthesia and sedation in the beach chair position. Whereas hypotension was frequent, cerebral desaturation was uncommon, occurring in $10 \%$ of patients [5]. Yet, a similar study performed in patients under general anaesthesia showed a significant decrease in cerebral arterial saturation [6]. Ischemia during TSA provoked by decreased cerebral blood flow in the beach chair position can have severe side effects such as loss of vision or ophthalmoplegia [7], hypoxic cervical spinal cord injury [8], or even catastrophic ischemic brain damage [9]. Additionally, studies such as those by Trentman et al. have shown that patients who receive antihypertensive medication face significant 
increases of intraoperative hypotension, and correspondingly, more often require vasopressors when placed in the beach chair position [10]. Given the meagre evidence, the American Patient Safety Foundation acknowledges that "we do not have a user friendly, non-invasive method for defining the lower limit of acceptable blood pressure for any given patient" [11], and the lower limit of acceptable blood pressure remains unclear.

Mashour et al. recently pointed out that the classic perioperative spotlight on hemodynamic might trigger insufficient vigilance in anaesthesiologists with regard to a patient's neurological status [12]. Whereas cerebral oxygenation monitoring is an established method in cardiac surgery, its value in non-cardiac settings is only just about to be recognized. In a recent review about the application of near-infrared spectroscopy (NIRS) in non-cardiac surgery Moerman and De Hert concluded "NIRS offers noninvasive monitoring of cerebral ...oxygenation in a wide range of clinical scenarios" [13]. Salazar et al. reported a $18 \%$ incidence of cerebral desaturation events in patients during shoulder surgery in beach chair position [14]. Regional cerebral tissue oxygen saturation $\left(\mathrm{SrcO}_{2}\right)$ may help to detect cerebral ischemia during procedures in beach chair position [15]. Which device to monitor $\mathrm{SrcO}_{2}$ in specific clinical contexts such as surgeries in beach chair position remains to be evaluated [16]. However, if such devices are not available, MAP remains a reliable measure for monitoring [15].

Going forward, a rational use of arterial lines for blood pressure monitoring during TSA will become of essence, given both the increasing economic and medico-legal pressures on health care providers. Where randomized controlled study designs are unavailable, logistic regression models of outcome data as presented by Gabriel et al. can provide insight in nationwide patterns and, thus, support clinical judgment. The results presented by Gabriel et al. are especially exciting because anaesthesiologists face multiple uncertainties when caring for patients positioned in beach chair. After studying the numbers reported in Figure 3 of their paper, you might wish to ask your junior resident to place an arterial line preoperatively in some older patients.

In their analysis of a large data set, Gabriel et al. shed light on the perioperative care for TSA patients in the U.S. It appears that few patients receive invasive blood pressure monitoring despite the fact that placement of an arterial line permits better monitoring of MAP and, thus, cerebral blood flow. Whereas, invasive cardiac parameters might be absent in many settings, the authors call for carefully considering preoperatively available data such as patient age, cardiac comorbidities, and foreseen longer surgeries as a guide for installation of invasive blood pressure monitoring. Albeit the authors examined outcomes for a specific operation, it appears feasible to directly extend their recommendations to any procedure in the upper extremity performed in the beach chair position.

\section{References}

1. McCall ML. Cerebral circulation and metabolism in toxemia of pregnancy; observations on the effects of veratrum viride and apresoline (1-hydrazinophthalazine). Am J Obstet Gynecol. 1953;66(5):1015-30.

2. Drummond JC. The lower limit of autoregulation: Time to revise our thinking? Anesthesiology. 1997;86(6):1431-3.

3. Moyer JH, Morris G. Cerebral hemodynamics during controlled hypotension induced by the continuous infusion of ganglionic blocking agents (hexamethonium, pendiomide and arfonad). J Clin Investig. 1954;33(8):1081-8. doi:10.1172/jci102980.

4. Gabriel RA, Beverly A, Dutton RP, Urman RD. Patterns of intraarterial blood pressure monitoring for patients undergoing total shoulder arthroplasty under general anesthesia: a retrospective analysis of 23,073 patients. J Clin Monit Comput. 2016. doi:10. 1007/s10877-016-9939-0.

5. Yadeau JT, Liu SS, Bang H, Shaw PM, Wilfred SE, Shetty T, Gordon M. Cerebral oximetry desaturation during shoulder surgery performed in a sitting position under regional anesthesia. Can J Anaesth. 2011;58(11):986-92. doi:10.1007/s12630-0119574-7.

6. Lee JH, Min KT, Chun YM, Kim EJ, Choi SH. Effects of beachchair position and induced hypotension on cerebral oxygen saturation in patients undergoing arthroscopic shoulder surgery. Arthrosc J Arthrosc Relat Surg. 2011;27(7):889-94. doi:10.1016/ j.arthro.2011.02.027.

7. Bhatti MT, Enneking FK. Visual loss and ophthalmoplegia after shoulder surgery. Anesth Analg. 2003;96(3):899-902.

8. Hindman BJ, Palecek JP, Posner KL, Traynelis VC, Lee LA, Sawin PD, Tredway TL, Todd MM, Domino KB. Cervical spinal cord, root, and bony spine injuries: a closed claims analysis. Anesthesiology. 2011;114(4):782-95. doi:10.1097/ALN. 0b013e3182104859.

9. Pohl A, Cullen DJ. Cerebral ischemia during shoulder surgery in the upright position: a case series. J Clin Anesth. 2005;17(6):463-9. doi:10.1016/j.jclinane.2004.09.012.

10. Trentman TL, Fassett SL, Thomas JK, Noble BN, Renfree KJ, Hattrup SJ. More hypotension in patients taking antihypertensives preoperatively during shoulder surgery in the beach chair position. Can J Anaesth. 2011;58(11):993-1000. doi:10.1007/s12630011-9575-6.

11. Lee L, Caplan R. APSF workshop: cerebral perfusion experts share views on management of head-up cases. APSF Newsl. 2009;24:46-8.

12. Mashour GA, Woodrum DT, Avidan MS. Neurological complications of surgery and anaesthesia. $\mathrm{Br} \mathrm{J}$ Anaesth. 2015;114(2):194-203. doi:10.1093/bja/aeu296.

13. Moerman A, De Hert S. Cerebral oximetry: The standard monitor of the future? Curr Opin Anaesthesiol. 2015;28(6):703-9. doi:10. 1097/aco.0000000000000256.

14. Salazar D, Sears BW, Aghdasi B, Only A, Francois A, Tonino P, Marra G. Cerebral desaturation events during shoulder arthroscopy in the beach chair position: patient risk factors and neurocognitive effects. J Shoulder Elb Surg Am Shoulder Elb Surg. 2013;22(9):1228-35. doi:10.1016/j.jse.2012.12.036. 
15. Kocaoglu B, Ozgen SU, Toraman F, Karahan M, Guven O. Foreseeing the danger in the beach chair position: Are standard measurement methods reliable? Knee Surg Sports Traumatol Arthrosc. 2015;23(9):2639-44. doi:10.1007/s00167-014-3090-6.
16. Scheeren TW, Bendjelid K. Journal of clinical monitoring and computing 2014 end of year summary: near infrared spectroscopy (NIRS). J Clin Monit Comput. 2015;29(2):217-20. doi:10.1007/ s10877-015-9689-4. 